\title{
Uncertain relevance of gastric colonization in the seriously ill *
}

\author{
J. F. Cade, E. McOwat, R. Siganporia, C. Keighley, J. Presneill and V. Sinickas \\ Intensive Care Unit and Microbiology Department, Royal Melbourne Hospital, Victoria, Australia
}

Received: 4 July 1991; accepted: 7 April 1992

\begin{abstract}
We have studied the temporal relationship for the same micro-organisms between gastric colonization and both nasopharyngeal colonization and major clinical infections in 100 consecutive, long-stay, intensive care patients. $67 \%$ of patients developed positive gastric cultures, mainly with aerobic Gram-negative bacilli and C. albicans; $33 \%$ developed positive nasopharyngeal cultures with similar organisms, but in only $8 \%$ was the same organism previously cultured from the stomach; $48 \%$ of patients developed infections, mainly respiratory, but commonly with different organisms. The presence of a positive gastric culture was not associated with gastric $\mathrm{pH}$, bleeding, severity of illness, or mortality. The results fail to confirm that an ascending migration of organisms from the stomach is frequent or that there is a relationship between gastric colonization and clinical infections. Firm therapeutic recommendation in these areas may be premature.
\end{abstract}

Key words: Gastric colonization - Nosocomial infections - Gastric acid - Selective decontamination

Gastric stress ulceration with consequent bleeding was a major complication of serious illness in previous years and carried substantial morbidity and mortality $[1,2]$. Stress ulcer prophylaxis has therefore been practised routinely in such patients, and clinicians have commonly used a parenteral $\mathrm{H} 2$ antagonist for this purpose [1-3]. Either because of this or because of other factors, gastric stress ulceration and haemorrhage have become uncommon problems in the seriously ill [2].

More recently, however, it has been suggested that such forms of stress ulcer prophylaxis may contribute to an increased incidence of nosocomial infections, particularly pneumonia. This sequence of infection has been considered to commence with gastric colonization, par-

\footnotetext{
* Supported by the Victor Hurley Medical Research Foundation, Royal Melbourne Hospital, Australia
}

ticularly with Gram-negative aerobic bacilli, due to loss of antimicrobial protection afforded by gastric acidity and consequent proximal migration of enteric bacteria from the large intestine [4-8]. Subsequent nasopharyngeal colonization occurs and later nosocomial pneumonia, septicaemia, perhaps infections elsewhere and possibly multiorgan failure [9-12].

Selective decontamination of the digestive tract (SDD, or selective parenteral and enteral antibiotic regimen, SPEAR) has been reported to interrupt this process by preventing gastric and nasopharyngeal colonization [11, $13-17]$. However, this a costly and messy technique, and other prophylactic strategies may be both more logical and more practical, depending on whether gastric stress ulceration is or is not still a potential problem, and whether its current prophylaxis is or is not harmful.

The present study was designed to provide information which would guide selection among these options for further formal study. The incidence and type of gastric colonization were studied in a large consecutive group of long-stay, critically ill patients, and the results were related both quantitatively and temporally with nasopharyngeal colonization, infectious complications, gastric $\mathrm{pH}$ and bleeding, and the patient's underlying conditions.

\section{Patients and methods}

\section{Patients}

The patients studied were consecutive admissions to a general Intensive Care Unit (ICU) who were critically ill and expected to stay for at least one week (i.e. defined for the purpose of this study as long-stay). This group of patients was considered to be the most scientifically efficient to study because the anticipated high rate of colonization and of infection would give the greatest chance of demonstrating the relationship sought and would reduce the likelihood of a false-negative result.

The further criteria for entry were the presence of a nasogastric tube and the routine administration of $\mathrm{H} 2$ antagonist medication (ranitidine $50 \mathrm{mg}$ IV b.i.d. in a standard dose in all patients). The study did not entail any new patient procedures or treatment.

The only criteria for exclusion were current haematemesis, known upper gastrointestinal tract pathology or enteral feeding (thus, the patients studied had gastrointestinal ileus and were parenterally fed as 
soon as feeding was clinically indicated). Patients were withdrawn from the study when enteral feeding commenced, as this could affect the inter-relationships among gastric stasis, stress ulceration and colonization. Patients were not withdrawn if they in fact stayed for less than one week, so that an intention-to-treat format could be preserved which might form a basis for any therapeutic trial that might follow.

\section{Procedure}

Surveillance cultures of gastric aspirate and nasopharyngeal swabs were made thrice weekly for the duration of admission, as reported by others [16]. The presence of enteric Gram-negative aerobic bacilli, methicillinresistant S. aureus (MRSA) and C. albicans were specifically sought. The methods comprised standard techniques of direct plating of samples onto blood agar, identification of the organisms sought, semiquantitative determination of orgnism numbers and antibiotic susceptibility testing $[16,18]$.

Diagnostic cultures were performed on usual clinical grounds in cases of respiratory or other infections for the presence and identification of pathogens. In particular, the temporal relationships of cultures of the same micro-organism from different sites were recorded.

The criteria for the diagnosis of nosocomial or secondary infection were according to CDC definitions [19]. The diagnosis of communityacquired or primary infection required that it be present either on, or within $48 \mathrm{~h}$ of, admission to ICU. The criteria for the diagnosis of colonization were positive results from the surveillance cultures and referred only to the stomach or throat.

Gastric pH (using pH strips, Merck, Darmstadt, Germany) and bleeding (using Gastroccult slides, Smith Kline Diagnostics, California, USA) were measured on the same aspirate that was cultured. Patient demographic data were recorded, including underlying condition, severity of illness, duration of ICU stay, presence of multi-organ failure and outcome. Severity of illness was assessed using the severity of illness class [20] and the therapeutic intervention scoring system (TISS) [21]. Multiorgan failure (MOF) was defined by usual criteria [22].

\section{Analysis}

Statistical analyses were by $\mathrm{X}^{2}$-test for discontinuous variables and unpaired $t$-test for continuous variables using conventional levels of probability. For continuous variables which were not normally distributed, normalization was achieved by transformation.

\section{Ethics}

The study was approved by the Hospital's Board of Medical Research.

\section{Results}

There were 100 consecutive patients studied during a 12 -month period. This represented $11 \%$ of all admissions to the ICU during this time, since the average duration of stay of all patients was 4.0 days and nearly $90 \%$ of admissions were of less than one week in duration.

The patient details are shown in Table 1 and the microbiological details in Table 2 . Of the patients $59 \%$ were men, the average age was 63 years (range 18-87 years), the average stay in hospital before ICU admission was 4.7 days (range $0-45$ days) and the average ICU stay was 16.2 days (range $2-75$ days). Of the patients $98 \%$ were mechanically ventilated, $74 \%$ had multi-organ failure, $89 \%$ had a severity of illness class of 4 (the remainder had class 3 ), the average TISS score was 62 (range 25-100) and the mortality was $54 \%$. The average gastric $\mathrm{pH}$ was 4.4 (range $(2-7.5)$ and the average lowest gastric $\mathrm{pH}$ was 3.6 (range 1-7.5). All patients had occult gastric bleeding, which was slight in $24 \%$, mild in $32 \%$ and moderate in $33 \%$; in 6 patients gastric bleeding was macroscopically visible, and in one patient it was clinically significant.

Patients with positive and negative gastric cultures were comparable (Table 3), except that positive gastric cultures were strongly associated with positive throat cultures $(p=0.0001)$ and with the duration of ICU stay $(p=0.0001)$. The duration of ICU stay was also strongly associated with the development of infection $(p=0.0001)$. An average of 3.6 gastric and throat cultures were taken per patient, with the average number of positive gastric cultures being 2.2 (range $0-21$ ) and of positive throat cultures being 1.2 (range $0-11$ ). The average time after ICU admission until cultures became positive was 4.9 days (range 1-21 days) for gastric cultures and 6.2 days (range $1-26$ days) for throat cultures.

The micro-organisms isolated from gastric and throat cultures were substantially similar, as shown in Table 4 , although in accord with previously reported studies [16] detailed typing was not done.

Approximately half of the patients $(48 \%)$ developed clinically significant infection, mostly respiratory but often at other sites. The average time after ICU admission until the diagnosis of infection was 5.6 days (range $1-13$ days). The relationship of the gastric culture result to these infections is shown in Table 5, in which it can be seen that there was no significant association between the presence of a positive gastric culture and the development of infection. Moreover, the micro-organisms involved in infections were substantially different from those cultured from the stomach or throat (Table 6).

For the same micro-organisms, a positive gastric culture preceded a positive throat culture in 15 patients ( $25 \%$ of those with positive throat cultures), but a positive throat culture preceded a positive gastric culture in seven patients $(10 \%$ of those with positive gastric cultures). In a further 19 patients, the same micro-organism appeared simultaneously in both gastric and throat cultures. A positive gastric or throat culture preceded an infection with the same micro-organism in eight patients (17\% of patients with infections) with infections in a total of 15 sites, with respiratory and urinary tract infections being of equal frequency in this latter regard ( 6 each): the stomach was first involved in 7 infections (in 3 patients), and the throat in 4 infections (in 2 patients); in a further 4 infections with the same micro-organism (in 3 patients), the stomach and throat were involved simultaneously.

\section{Discussion}

The high incidence of gastric colonization in seriously ill patients shown in the present study is in accord with previous reports, except that the present incidence is generally higher $[6-8,11]$. This may be because the present study concerned the sickest and longest staying intensive care patients, who would presumably be most likely to become colonized.

In brief, $67 \%$ of patients developed positive gastric cultures. This occurred on average on the fifth day of admission to the ICU or tenth day of hospital admission, 
Table 1. Patient details

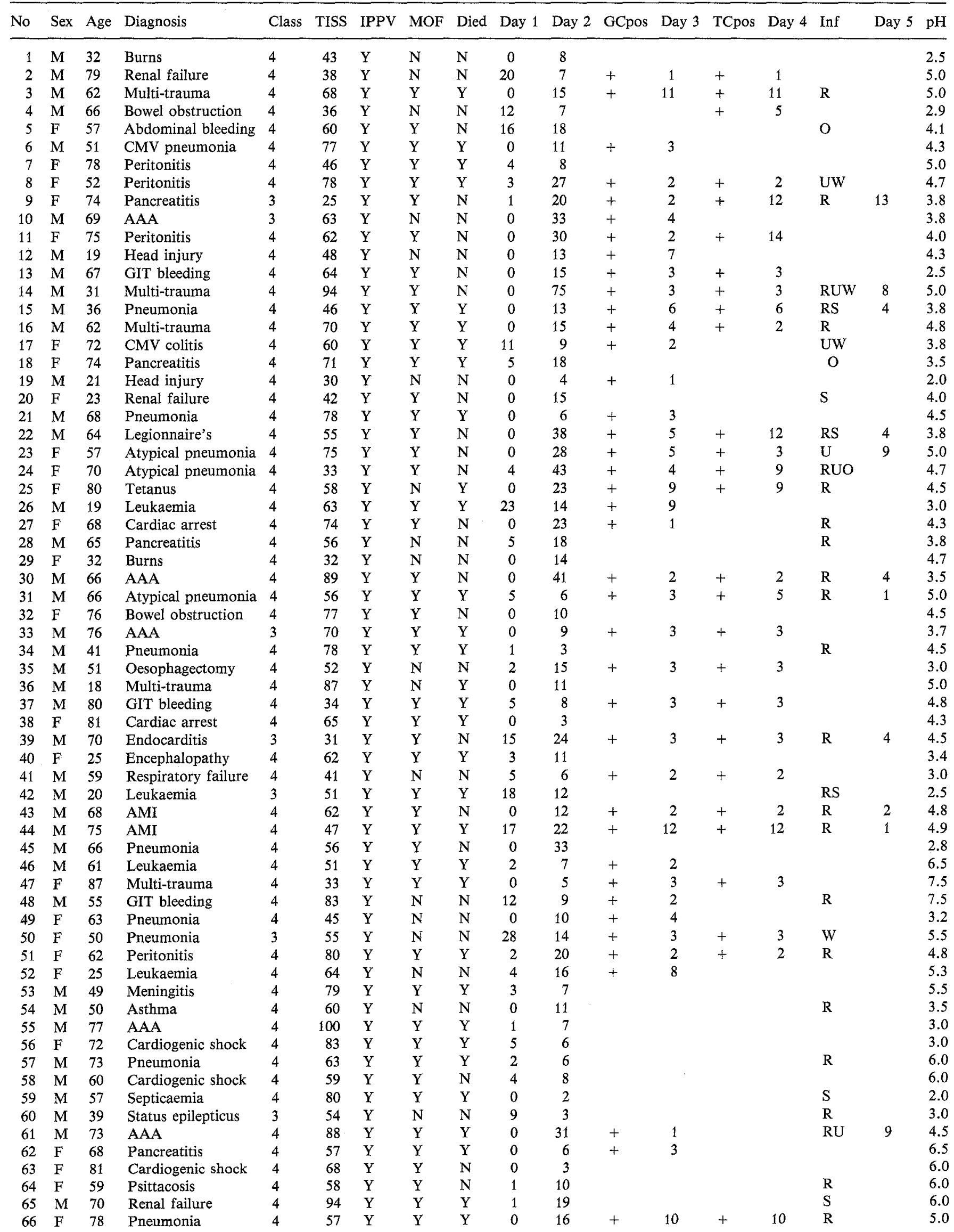


Table 1 (continued)

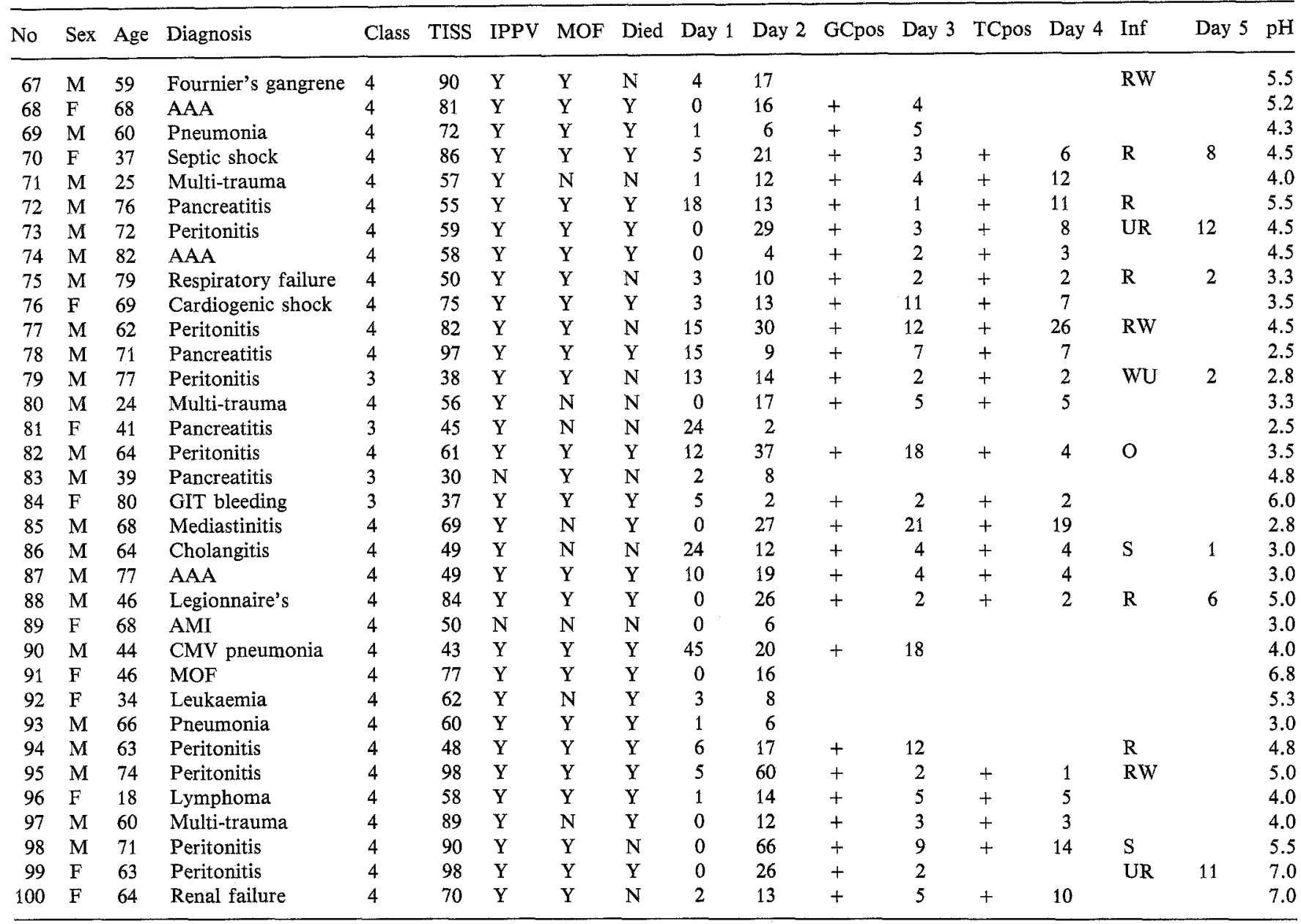

For Diagnosis, CMV is cytomegalovirus, AAA is abdominal aortic aneurysm, GIT is gastrointestinal tract, AMI is acute myocardial infarction and MOF is multi-organ failure; IPPV is intermittent positive pressure ventilation (i.e. mechanical ventilation); Day 1 is no. of days in hospital before admission to ICU; Day 2 is no. of days in ICU; GCpos is positive gastric culture; Day 3 is ICU day of first positive gastric culture; TCpos is positive throat culture; Day 4 is ICU day of first positive throat culture; Inf is infection with $\mathrm{R}=$ respiratory, $\mathrm{U}=$ urinary, W $=$ wound, $\mathrm{S}=$ septicaemia and $\mathrm{O}=$ other; Day 5 is first ICU day of infection if same organism as gastric or throat culture; $\mathrm{pH}$ is average gastric $\mathrm{pH}$

Table 2. Microbiological details

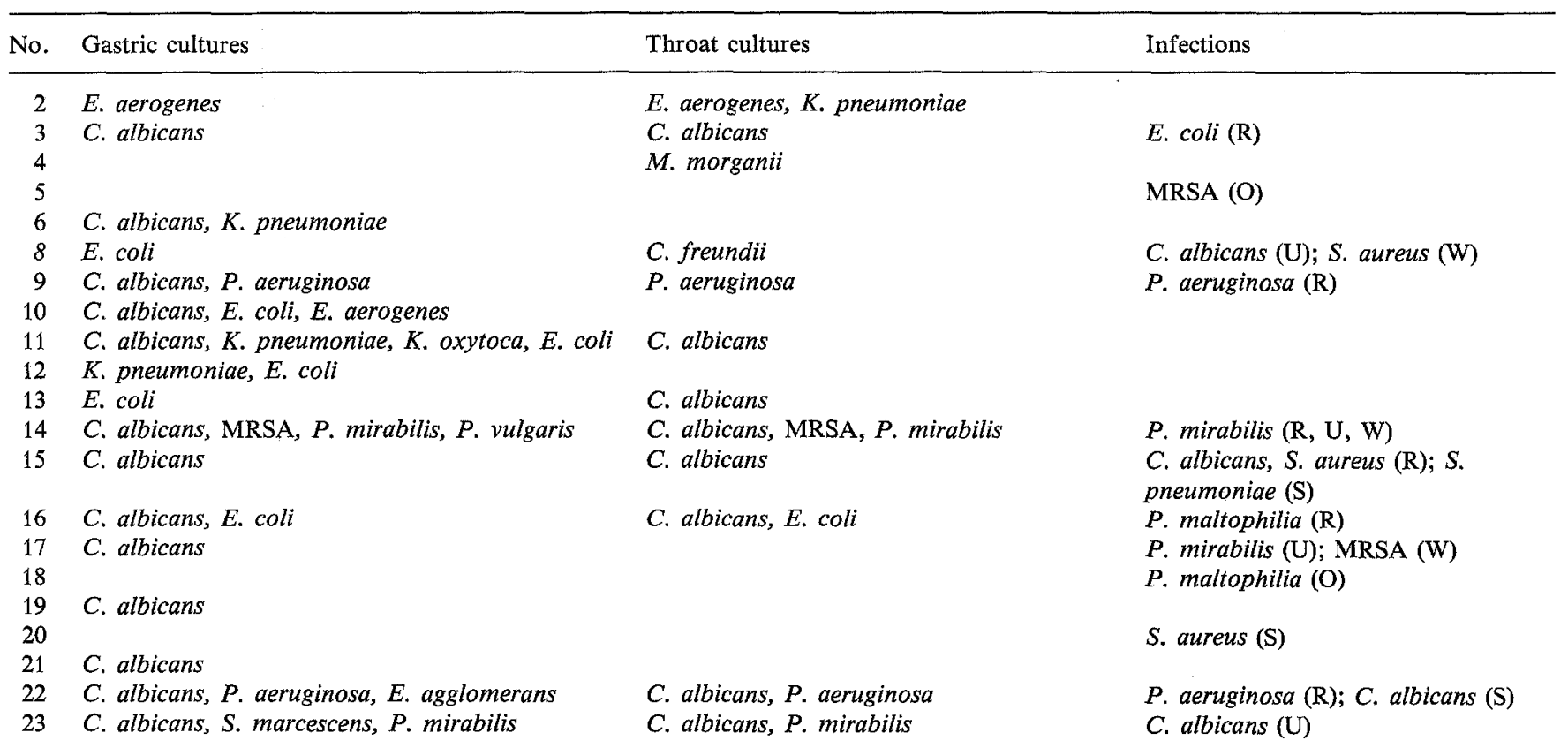


Table 2 (continued)

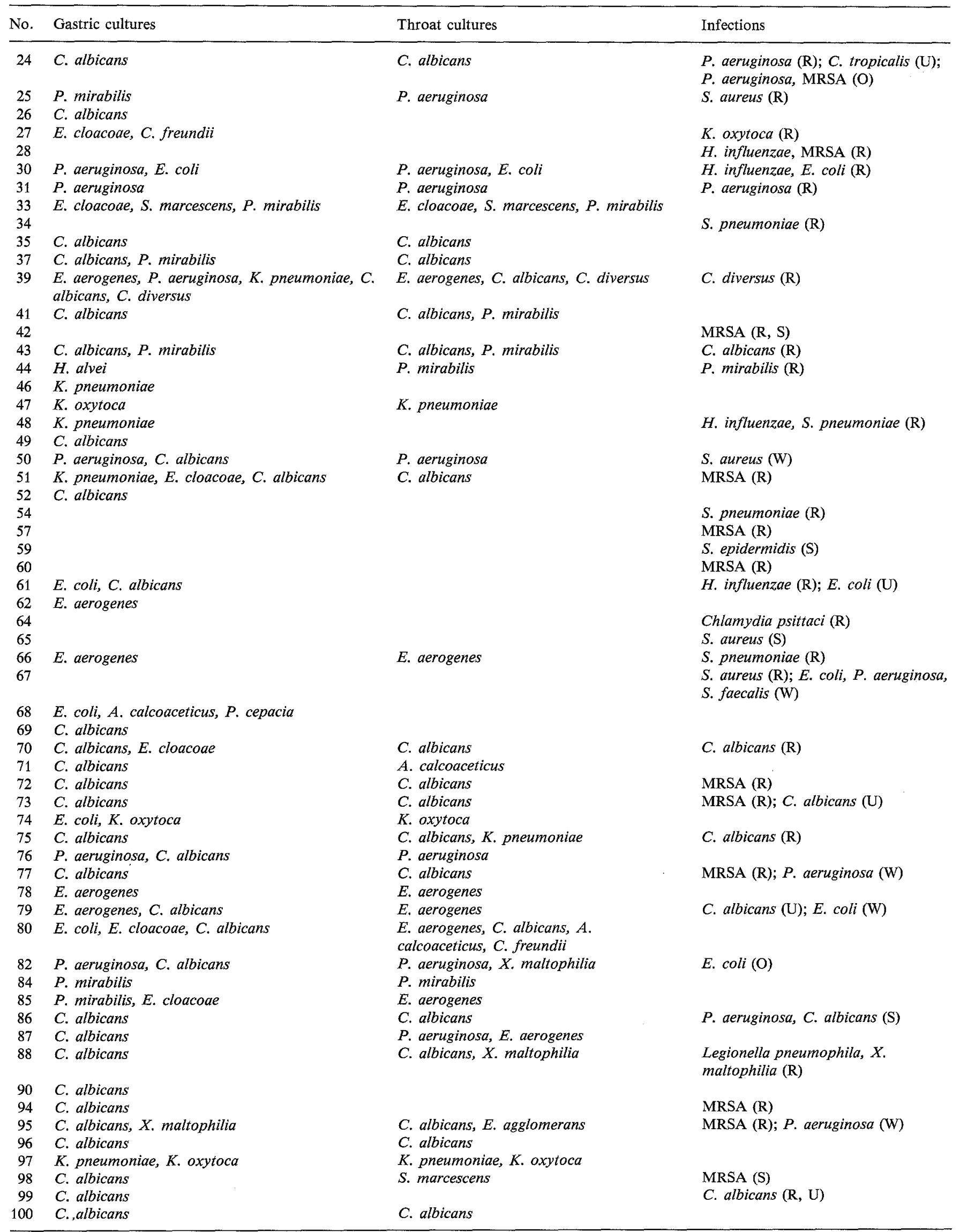

No. is patient number from Table 1. For Infections, abbreviations are as in Table 1 
Table 3. Patient details in relation to gastric colonization

\begin{tabular}{llll}
\hline & \multicolumn{2}{l}{ Gastric Culture } & \multirow{2}{l}{$p$} \\
\cline { 2 - 3 } & Positive & Negative & \\
\hline Total & 67 & 33 & \\
Sex (M/F, \%) & $67 / 33$ & $55 / 45$ & NS \\
Age (yr) & $61(17)$ & $54(19)$ & NS \\
Class & $3.9(0.3)$ & $3.9(0.3)$ & NS \\
TISS & $62(18)$ & $63(18)$ & NS \\
IPPV (\%) & 100 & 94 & NS \\
MOF (\%) & 76 & 70 & NS \\
Mortality (\%) & 57 & 48 & NS \\
Hospital Days (pre-ICU) & $5.1(8.5)$ & $3.8(5.8)$ & NS \\
ICU Days & $19.2(14.2)$ & $10.1(6.5)$ & 0.0001 \\
Positive Throat Cultures (\%) & 72 & 3 & 0.0001 \\
Infections (\%) & 52 & 39 & NS \\
pH (average) & $4.4(1.2)$ & $4.2(1.3)$ & NS \\
pH (lowest) & $3.6(1.5)$ & $3.8(1.4)$ & NS \\
pH (average >4) (\%) & 67 & 55 & NS \\
\hline
\end{tabular}

Values are mean with standard deviation in brackets, where indicated

Table 4. Micro-organisms isolated from gastric and throat cultures

\begin{tabular}{lcc}
\hline Micro-organism & Gastric & Throat \\
\hline E. coli & 11 & 2 \\
K. pneumoniae & 8 & 4 \\
K. oxytoca & 4 & 2 \\
$P$. aeruginosa & 8 & 9 \\
$P$. cepacea & 1 & 0 \\
$P$. mirabilis & 8 & 7 \\
$P$. vulgaris & 1 & - \\
E. aerogenes & 7 & 8 \\
E. cloacoae & 6 & 1 \\
E. agglomerans & 1 & 1 \\
S. marcescens & 2 & 2 \\
H. alvei & 1 & - \\
C. freundii & 1 & 2 \\
C. diversus & 1 & 1 \\
$X$. maltophilia & 1 & 2 \\
A. calcoaceticus & 1 & 2 \\
M. morganii & - & 1 \\
C. albicans & 46 & 26 \\
S. aureus (methicillin-resistant) & 1 & 1 \\
No. positive isolates & 109 & 71 \\
No. patients with positive isolates & 67 & 49 \\
\hline
\end{tabular}

Table 5. Relation of gastric cultures to infections

\begin{tabular}{lll}
\hline & \multicolumn{2}{l}{ Gastric culture } \\
\cline { 2 - 3 } & Positive & Negative \\
\hline Respiratory & 27 & 8 \\
Urinary & 9 & 0 \\
Wound & 7 & 1 \\
Septicaemia & 4 & 4 \\
Other & 2 & 2 \\
All patients with infection & $35(52 \%)$ & $13(39 \%)$ \\
All patients & 67 & 33 \\
\hline
\end{tabular}

Comparison of patients with positive and negative gastric cultures showed no statistically significant difference in infection, either for all infections or for type of infection
Table 6. Micro-organisms isolated in nonocomial infections

\begin{tabular}{|c|c|c|c|c|c|}
\hline Micro-organism & Respiratory & Urinary & Wound & Septicaemia & Other \\
\hline E. coli & 2 & 1 & 2 & & 1 \\
\hline$P$. aeruginosa & 4 & & 3 & 1 & 1 \\
\hline P. mirabilis & 2 & 2 & 1 & & \\
\hline P. maltophilia & 1 & & & & 1 \\
\hline K. oxytoca & 1 & & & & \\
\hline C. diversus & 1 & & & & \\
\hline C. tropicalis & & 1 & & & \\
\hline$X$. maltophilia & 1 & & & & \\
\hline C. albicans & 5 & 5 & & 2 & \\
\hline $\begin{array}{l}\text { S. aureus } \\
\text { (methicillin- } \\
\text { resistant) }\end{array}$ & 10 & & 1 & 2 & 2 \\
\hline S. aureus & 3 & & 2 & 2 & \\
\hline S. epidermidis & & & & 1 & \\
\hline S. pneumoniae & 1 & & & & \\
\hline S. faecalis & & & 1 & & \\
\hline H. influenzae ${ }^{\mathrm{a}}$ & 4 & & & & \\
\hline Total & 35 & 9 & 10 & 8 & 5 \\
\hline
\end{tabular}

a Indicates that this micro-organism would not have been isolated by the surveillance methods used for gastric and nasopharyngeal specimens

although some cultures were positive on the first day of hospital admission even in previously well patients. All positive cultures had occurred by three weeks of ICU admission, and conversely negative cultures were uncommon in patients staying longer than three weeks. More than half the isolates $(56 \%)$ were of Gram-negative aerobic bacilli and most of the rest were of C.albicans; the isolation of MRSA was uncommon $(<1 \%)$.

Patients with positive gastric cultures were not demographically different from those with negative cultures. In particular, there was no difference in gastric $\mathrm{pH}$, whether analyzed as average, lowest or proportion above or below 4.0. Specifically, there was no tendency for gastric colonization to be associated with a higher gastric $\mathrm{pH}$. It was of interest that gastric $\mathrm{pH}$ was mostly above 4.0 , even though a standard rather than titrated dose of ranitidine was given routinely. This may be the explanation for the unexpected lack of relationship between gastric $\mathrm{pH}$ and colonization, since discrimination could be lost as the entire range of observed $\mathrm{pH}$ values was elevated above normal.

The striking differences between patients with positive and negative gastric cultures related to duration of ICU stay and to nasopharyngeal colonization. The patients with positive gastric cultures had an average duration of stay twice as long as those with negative cultures. While this could possibly be related to the more numerous cultures that were inevitably taken during longer admissions, it would seem more likely that the risk of gastric colonization was directly related to the duration of admission. Further, as indicated above, gastric colonization was almost universal by 3 weeks.

Gastric colonization was very strongly associated with nasopharyngeal colonization $(p=0.0001)$. However, gastric colonization was more frequent than nasopharyngeal colonization ( $67 \%$ and $49 \%$, respectively), so that while 
nasopharyngeal colonization was virtually always associated with gastric colonization, the converse could not of course apply. While the same types of micro-organisms were involved at both sites, the temporal relationship between positive cultures at the two sites for the same micro-organism failed to support the suggested sequence of gastric colonization frequently leading to nasopharyngeal colonization [4-8]. Thus, only $25 \%$ of positive throat cultures were preceded by a positive gastric culture for the same micro-organgism. Moreover, $10 \%$ of positive gastric cultures were preceded by a positive throat culture for the same micro-organism. These are maximum estimates of linkage and would decrease if more detailed bacterial typing than would be appropriate for general surveillance showed that micro-organisms seemingly the same were in fact different. On the other hand, these estimates of linkage could increase if more frequent sampling than would be appropriate for general surveillance showed that the seemingly simultaneous occurrence of micro-organisms was in fact sequential. This could apply in up to 19 patients with gastric and throat colonization but in only three patients with infections. On balance, therefore, while the data are consistent with an ascending movement of micro-organisms in some patients, they suggest a descending movement in other patients and no directional transfer in most patients.

Furthermore, the relationship between gastric (or nasopharyngeal) colonization and clinically significant infection was even more tenuous. Thus, despite the frequency of infection, which was found in nearly half the patients, the same micro-organism was isolated from a site of infection and from gastric or nasopharyngeal cultures in only $17 \%$ of patients with infections and these were equally divided between respiratory and urinary tract infections. This lack of relationship has been previously observed by some [23] but not by others [9-12,24]. Moreover, the types of micro-organisms involved in infections were in general substantially different from those identified in the surveillance cultures of gastric or nasopharyngeal specimens. The finding of Pseudomonas as a more common Gram-negative aerobic bacillus in respiratory infections $(33 \%)$ than in nasopharyngeal cultures $(20 \%)$ is in accord with previous reports (25), even though the major risk factor of inadequate nutrition was carefully attended to in the present study. A high incidence was also found of MRSA in respiratory infections $(25 \%)$ compared with nasopharyngeal or gastric cultures ( $1 \%$ each), suggesting that the route of entry of this micro-organism was most likely directly via the endotracheal tube.

The present study involved a large group of the sickest and longest staying ICU patients, in whom it might be considered that the potential relevance of gastric colonization could be most efficiently explored. However, although gastric colonization was frequent, the results have failed to confirm a substantial ascending migration of micro-organisms to the nasopharynx or a significant relationship with clinically important infection (particularly at respiratory, urinary, wound and systemic sites). Thus, the rationale to adopt SDD (or SPEAR) in such patients appears to us to be uncertain and alternative strategies may be more attractive. For example, perhaps sucralfate could be used for gastric stress ulceration instead of an $\mathrm{H} 2$ antagonist, so that gastric $\mathrm{pH}$ could be preserved at a lower and more normal level $[26,27]$. However, we could find no relationship between gastric $\mathrm{pH}$ and colonization, so that the level of gastric $\mathrm{pH}$ may not be as relevant as has traditionally been considered. Even more importantly, the risk of gastric stress ulceration may no longer be as high as it once was, and prophylaxis of any sort may be unnecessary as a routine measure [2]. It seems to us that the evidence to date is too incomplete to a make a firm therapeutic recommendation in these areas, and clearly more information is required.

\section{References}

1. Geus WP, Lamers CBHW (1969) Prevention of stress ulcer bleeding. A review. Scand J Gastroenterol 25 [Suppl 178]:32-41

2. Reines HD (1990) Stress ulcer - gone the way of polio and smallpox? In: Lumb PD, Shoemaker WC (eds) Critical care - state of the art. SCCM, Fullerton, pp 259-269

3. Priebe HJ, Skillman JJ, Bushnell JS, Long PC, Silen W (1989) Antacid versus cimetidine in preventing acute gastrointestinal bleeding. A randomized trial in 75 critically ill patients. $N$ Engl $\mathbf{J}$ Med 402:426-430

4. Atherton TS, White DJ (1978) Stomach as source of bacteria colonising the respiratory tract during artificial ventilation. Lancet II:968-969

5. Moulin GC du, Paterson DG, Hedley-Whyte J, Lisbon A (1982) Aspiration of gastric bacteria in antacid-treated patients: a frequent cause of postoperative colonization of the airway. Lancet I:242-245

6. Hillman KM, Riordan T, O'Farrell SM, Tabaqchal S (1982) Colonization of gastric contents in critically ill patients. Crit Care Med $10: 444-447$

7. Pingleton SK (1991) The gut and infection. Pulmonary perspectives. ACCP 8:1-4

8. Garvey BM, McCambley JA, Tuxen DV (1989) Effects of gastric alkalinization on bacterial colonization in critically ill patients. Crit Care Med 17:211-216

9. Johanson WG, Pierce AK, Sanford JP (1969) Changing pharyngeal bacterial flora of hospitalized patients. $N$ Engl $J$ Med 281:1137-1140

10. Craven DE, Kunches LM, Kilinsky V, Lichtenberg DA, Make BJ, McCabe WR (1986) Risk factors for pneumonia and fatality in patients receiving continuous mechanical ventilation. Am Rev Respir Dis 133:792-796

11. van Saene HKF, Stoutenbeek CP, Lawin P, Ledingham IM (eds) (1989) Infection control in intensive care units by selective decontamination. Springer, Berlin

12. Mason CM (1991) The pathogenesis and prevention of nosocomial pneumonia. Crit Care Rep 2:145-157

13. Stoutenbeek CP, van Saene HKF, Miranda DR, Zandstra DF, Langrehr D (1986) Nosocomial gram-negative pneumonia in critically ill patients. A 3-year experience with a novel therapeutic regimen. Intensive Care Med 12:419-423

14. Stoutenbeek CP, van Saene HKF, Miranda DR, Zandstra DF, Langrehr D (1987) The effect of oropharyngeal decontamination using topical non-absorbable antibiotics on the incidence of nosocomial respiratory tract infections in multiple trauma patients. J Trauma 27:357-364

15. van Uffelen R, Rommes JH, van Saene HKF (1987) Preventing lower airway colonization and infection in mechanically ventilated patients. Crit Care Med 15:99-102

16. Ledingham IM, Alcock SR, Eastaway AT, McDonald JC, McKay IC; Ramsay G (1988) Triple regimen of selective decontamination of the digestive tract, systemic cefotaxime, and microbiological sur- 
veillance for prevention of acquired infection in intensive care. Lancet I:785-790

17. van Saene HKF, Hart CA (1991) Selective decontamination: are microbiologists more resistant than microbes? Host 3:6-9

18. Lennette EH, Balows A, Hausler WJ, Shadomy HJ (eds) (1985) Manual of clinical microbiology, 4th edn. American Society for Microbiology, Washington DC

19. Garner JS, Jarvis WR, Emori TG, Horan TC, Hughes JM (1988) CDC definitions for nosocomial infections, 1988. Am J Infect Control 16:128-140

20. Cullen DJ (1977) Results and costs of intensive care. Anesthesiology 47:203-216

21. Cullen DJ, Civetta JM, Briggs BA, Ferrara LC (1974) Therapeutic intervention scoring system: a method for quantitative comparison of patient care. Crit Care Med 2:57-60

22. Cerra FB, Negro F, Eyer S (1990) Multiple organ failure syndrome: patterns and effect of current therapy. In: Vincent JL (ed) Update in intensive care and emergency medicine, no. 10, Update 1990. Springer, Berlin, pp 22-31

23. Reusser P, Zimmerli, W, Scheidegger D, Marbet GA, Buser M, Gyr $\mathrm{K}$ (1989) Role of gastric colonization in nosocomial infections and endotoxaemia. J Infect Dis 160:414-421
24. Pugin J, Auckenthaler R, Lew DP, Suter PM (1991) Oropharyngeal decontamination decreases incidence of ventilator-associated pneumonia. JAMA 265:2704-2710

25. Niederman MS, Mantovani R, Schoch P, Papas J, Fein AM (1989) Patterns and routes of tracheobronchial colonization in mechanically ventilated patients. Chest 95:155-161

26. Driks MR, Graven DE, Celli BR, Manning M, Burke RA, Garvin GM, Kunches LM, Farber HW, Wedel SA, McCabe WR (1987) Nosocomial pneumonia in intubated patients given sucralfate as compared with antacids or histamine type 2 blockers. N Engl J Med $317: 1376-1382$

27. Tryba M (1987) Risk of acute stress bleeding and nosocomial pneumonia in ventilated intensive care unit patients; sucralfate versus antacids. Am J Med 83 [Suppl 3B]:117-124

Dr. J.F. Cade

Royal Melbourne Hospital

Victoria 3050

Australia 